at the end where they were situated. The initial distribution of excited atoms was almost certainly more complex, so that higher-order solutions with larger decay constants also have to be taken into account.

It is possible that a repetition of this type of experiment in a large vessel at a pressure sufficiently low to ensure uniformity of the discharge, but not so low as to make loss by diffusion overwhelmingly great, might allow the various processes involved to be sorted out, and hence give an experimental value for the quadrupole transition probability.

We are indebted to the mañager of the Research Laboratories of the General Electric Co., Ltd., Wembley, for the gift of the electrode tube.

K. G. Emeinéus

N. D. SAYERS

R. A. BAILEY

Queen's University,

Belfast.

May 17.

'Vegard, L., and Kvifte, G., Nature, 162, 967 (1948).

'Jenkins, H. G., Bowtell, J. N., and Strong, R. W., Nature, 163, 401 (1949).

${ }^{3}$ Herman, L. and R., Ann. Géophys., 1, 165 (1944).

- Pasternack, Astrophys. J., 92, 129 (1940).

5 Janin, J., Thesis, Paris (1946).

Jeans, J. H., "Kinetic Theory of Gases", Chapter 7 (Cambridge, 1940 ).

\section{A Linear Theory of Finite Strain}

Several papers have been published by Dr. K. H. Swainger in which he claims to have originated a linear theory of elasticity involving finite strain, and he has questioned, not only the approach of other workers in the same field, but also the classical infinitesimal theory, since these appear to be in conflict with his own ${ }^{1,2}$. The purpose of this communication is to show that this new theory is untenable, since a mathematical inconsistency enters at the very beginning.

Dr. Swainger bases his discussion of strain on the following ideas (for simplicity the two-dimensional case will be considered). Let the points $Q, Q^{\prime}$, and hence the element $d \mathbf{R}$, in the strained body correspond to $P, P^{\prime}$ and $d \mathbf{r}$, respectively, in the unstrained body.
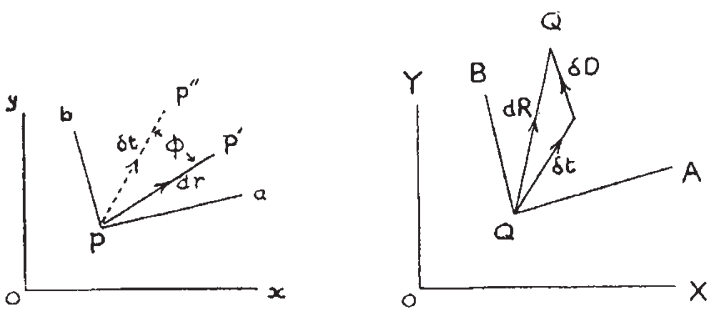

At $P$ there exists a unique pair of directions $P a$, $\mathrm{Pb}$ which are orthogonal and which transform into a pair of orthogonal directions $Q A, Q B$ in the strained body $^{3}$. Suppose that the axes $P a, P b$ are rotated about $P$ until they are parallel to $Q A, Q B$, carrying $d \mathbf{r}$ with them into a new position $\delta \mathbf{t}$. Then the infinitesimal straining displacement of $Q^{\prime}$ relative to $Q$ is defined by the relation

$$
\delta \mathbf{D}=d \mathbf{R}-\delta \mathbf{t} .
$$

For a point $S$ at a finite distance from $Q$ the straining displacement of $S$ relative to $Q$ is then defined as

$$
\mathbf{D}=\int_{Q}^{S} \delta \mathbf{D}=\int_{Q}^{S} d \mathbf{R}-\int_{Q}^{S} \delta \mathbf{t},
$$

the rotation described above being applied to each element $d \mathbf{r}$ of the path in the unstrained body which corresponds to $Q S$. In the subsequent theory this quantity $\mathbf{D}$ replaces the conventional displacement

$$
\mathbf{U}=\int_{Q}^{S} d \mathbf{R}-\int_{Q}^{S} d \mathbf{r}
$$

It is tacitly assumed throughout Dr. Swainger's theory that D has a meaning; that is, that the process employed in its derivation is independent of the particular path between $Q$ and $S$ in equation (2). This is, however, false. Let the 'local rotation' at $P$ necessary to bring the direction of $P a$ into coincidence with that of $Q A$ be denoted by $\varphi$, an angle which is in general a function of position. It does not follow, of course, that any arbitrary function can be chosen to represent $\varphi$, since such a choice may not be consistent with the physics of the problem. However, the non-integrability of $\delta \mathbf{D}$ will be shown without any restrictions on $\varphi$ whatsoever, beyond the assumption that it is a continuous one-valued function of position.

From equation (1), $\delta \mathbf{D}$ is a perfect differential only if $\delta \mathbf{t}$ is. Let $d \mathbf{r}$ have components $d x, d y$ parallel to the axes of a fixed reference system $O x y$. Then

$$
\begin{aligned}
& \delta t_{x}=d x \cos \varphi-d y \sin \varphi, \\
& \delta t_{y}=d x \sin \varphi+d y \cos \varphi,
\end{aligned}
$$

must be perfect differentials; that is, one-valued quantities $t_{x}$ and $t_{y}$ must exist such that

$$
\frac{\delta t_{x}}{\delta x}=\frac{\delta t_{y}}{\delta y}=\cos \varphi, \frac{\delta t_{x}}{\delta y}=-\frac{\varepsilon t_{y}}{\delta x}=-\sin \varphi .
$$

But these are the Cauchy-Riemann relations and are sufficient to ensure that $\tau=t_{x}+i t_{y}$ is an analytic function of $z=x+i y$, regular in the region under consideration.

Hence

$$
\frac{d \tau}{d z}=\frac{\delta t_{x}}{\delta x}+i \frac{\delta t_{y}}{\delta x}=\exp i \varphi
$$

is also a function of $z$, so that $\varphi$ must be a function of $z$. But $\varphi$ is a real quantity, and as such can only be a constant.

Hence the quantity D used in Dr. Swainger's theory has a meaning only for irrotational strain (apart from a possible rigid-body rotation which may also be superimposed). In these circumstances it is effectively equivalent to the conventional displacement defined in equation (3).

Imperial College of Science and Technology, London, S.W.7.

' Swainger, K. H., Nature, 164, 23 (1949).

Swainger, K. H., Nature, 165, 159 (1950).

s Love, A. E. H., "Mathematical Theory of Elasticity".

DR. Gordon describes a theory of 'straining' that is certainly not mine. Dr. Gordon merely discusses the rotation of an assembly of elemental rigid blocks (or the undeformed body) with initial vector diagonal $d \mathbf{r}$ and current diagonal $d \mathbf{t}$, and correctly finds that since the body is not deformable then it can only 